\title{
Extraction, enrichment, and in situ electrochemical detection on lab- on-a-disc; monitoring the production of a bacterial secondary metabolite
}

Andreasen, Sune Zoëga; Sanger, Kuldeep; Jendresen, Christian Bille; Nielsen, Alex Toftgaard; Emnéus, Jenny; Boisen, Anja; Zor, Kinga

\section{Published in:}

ACS Sensors

Link to article, DOI:

$10.1021 /$ acssensors.8b01277

Publication date:

2019

Document Version

Peer reviewed version

Link back to DTU Orbit

Citation (APA):

Andreasen, S. Z., Sanger, K., Jendresen, C. B., Nielsen, A. T., Emnéus, J., Boisen, A., \& Zor, K. (2019). Extraction, enrichment, and in situ electrochemical detection on lab- on-a-disc; monitoring the production of a bacterial secondary metabolite. ACS Sensors, 4(2), 398-405. https://doi.org/10.1021/acssensors.8b01277

\section{General rights}

Copyright and moral rights for the publications made accessible in the public portal are retained by the authors and/or other copyright owners and it is a condition of accessing publications that users recognise and abide by the legal requirements associated with these rights.

- Users may download and print one copy of any publication from the public portal for the purpose of private study or research.

- You may not further distribute the material or use it for any profit-making activity or commercial gain

- You may freely distribute the URL identifying the publication in the public portal 
Since the development of the first Micro Total Analysis System, ${ }^{1,2}$ an extensive number of articles have been published, reporting advances in the field of microfluidic devices. ${ }^{3-5}$ However, the commercialization of these platforms continues to be a challenge..$^{6-8}$ One of the major limiting factors when aiming to develop systems for reallife applications, and dealing with complex sample matrices. ${ }^{9}$ Components in the sample matrix can either interfere with the detection or cause fouling of the sensor, while sample heterogeneity could lead to inconsistency in detection. ${ }^{9,10}$ This issue can be solved either by coupling or directly integrating the sample pre-treatment with the sensor on the miniaturized platform. ${ }^{11-17}$

Centrifugal microfluidic devices also referred to as lab-ona-disc (LoD) systems, enable complex sample handling and assays where low sample and reagent volumes are needed. They facilitate automation, using mostly simple equipment/hardware and components (spinning motors, polymer discs, diodes, etc.) while keeping a small footprint of the final device. ${ }^{18,19}$ Thus, the field of centrifugal microfluidics provide promising tools for the development of advanced, yet cost-efficient, analytical devices, for instance within analysis of biological samples, or diagnostics. ${ }^{11,12,19-22}$ Although the principle of liquid handling is as simple as introducing centrifugal forces by rotating the LoD device, by clever design, a range of advanced sample handling procedures have been adapted and integrated on these platforms, including valving, mixing, metering, filtering, flow-switching, and centrifugation. ${ }^{15,18,23-25}$

In this work, we introduce a new, powerful, sample pretreatment tool to the centrifugal microfluidic tool-box based on supported liquid membrane (SLM) extraction. SLM extraction is a sample pretreatment technique used in analytical chemistry for purifying and enriching analytes directly from complex matrices, such as saliva, ${ }^{16}$ urine, ${ }^{26}$ or soil. ${ }^{27}$ SLM extraction and subsequent analyte detection are usually performed in two steps, ${ }^{28-30}$ however, automation of the extraction, followed by high performance liquid chromatography (HPLC) has been demonstrated. ${ }^{31}$ Nevertheless, such systems, even when automated, are dependent on experienced technicians to perform the analysis, and still require large consumption of eluents and solvents.

Electrochemical detection is particularly well suited for microfluidic integration as it can be miniaturized without compromising sensitivity, and is easily multiplexed. ${ }^{13,32-34}$ Furthermore, signals can be processed using conventional electronic components, ${ }^{35}$ and a variety of non-conductive materials, e.g, glass, ${ }^{36}$ plastic, ${ }^{15}$ or silicone ${ }^{37}$ can be used as a substrate for patterning the electrode.

Recently, we demonstrated that we can electrochemically measure $p$-Coumaric acid, a hydroxycinnamic acid (pHCA), produced by Escherichia coli (E.coli.), at the end point of the bioprocess. ${ }^{15}$ However, detection and quantification 
during production, especially at low concentration, proved to be challenging. This is due to the complexity of the sample, as well as the proximity of the redox potentials of the product (pHCA) and the substrate (Tyrosine (Tyr). ${ }^{38-}$ 40 Therefore, we developed a protocol for on-chip SLMbased extraction of pHCA from the bacterial supernatant, and highlighted the advantages and applicability of this extraction step when used in combination with off-chip surface enhanced Raman scattering-based detection. ${ }^{14}$

Here we report for the first time the integration of SLM extraction with in-situ electrochemical detection on a LoD platform. By integrating the electrochemical sensor directly below the extraction unit on the acceptor side, the analytical procedure is greatly simplified. Moreover, it enables the simultaneous clean-up, extraction, enrichment, and analyte detection, on the same platform. The eight SLM extraction and electrochemical detection units facilitate the full analysis from sample to answer. Besides being an efficient sample pre-treatment method, ${ }^{41}$ SLM extraction requires very low volume of organic solvents, and is thus an environmentally friendly, low-cost extraction method. ${ }^{42}$

As a case study, we evaluated the performance of a pHCA producing $E$. coli bacterial strain during a $24 \mathrm{~h}$ bioprocess by monitoring the production of pHCA over time. pHCA is a naturally occurring phenolic acid, with strong antioxidant properties, ${ }^{43}$ used both as a food additive, ${ }^{44}$ and as a precursor for the development of pharmaceuticals. ${ }^{45}$ Bacterial factories, such as the genetically modified E.coli expressing tyrosine ammonia-lyase (TAL), which converts Tyr to pHCA, is one approach for the production of this analyte ${ }^{46-48}$ in industrially relevant quantities. When screening for the best pHCA producing bacterial strains it is paramount to be able to distinguish pHCA from Tyr, as early as possible, in order to determine the yield of the bioprocess and cut down on both development time and cost. This is challenging since the two molecules are very similar, both in terms of optical properties and redox potentials ${ }^{38-40}$. Currently, the methods used to identify the best performing strains are either based on spectrophotometry $^{48}$, or classical chromatographic techniques, such as HPLC ${ }^{46}$ or thin-film chromatography. ${ }^{49}$ These methods, although highly accurate and precise, are slow, costly, laborious, and often require large amounts of organic solvents. All in all, the screening process of large number of candidate bacterial strains turns out to be by far the biggest bottleneck in the development cycle. ${ }^{50}$

\section{Experimental section}

Chemicals. Solutions containing pHCA and Tyr were made by diluting stock solutions of pHCA in ethanol $(50 \mathrm{mM}$ in 99\% ethanol) and Tyr in basic solution (5 mM Tyr in a $\mathrm{KOH}$ solution at $\mathrm{pH} 11$ ). The minimal M9 medium ${ }^{51}$ was used for bacterial culture containing $10 \mathrm{~g} \mathrm{~L}-1$ glucose, 2 $\mathrm{mM}$ Tyr, $1 \mathrm{mM}$ Isopropyl-beta-D-thiogalactoside and Wolfe's Vitamin solution purchased from ATCC®) (LGC Standards, UK) as described earlier. ${ }^{52}$ For cyclic voltammograms (CV), $10 \mathrm{mM}$ potassium ferro/ferricyanide was dissolved in phosphate buffered saline (PBS), pH 7.4, containing 10mM PB, 150 mM NaCl. pHCA standards for the calibration curve were prepared in Tris buffer (50 mM Tris, $150 \mathrm{mM} \mathrm{NaCl,} \mathrm{pH} \mathrm{7.6),} \mathrm{which} \mathrm{was} \mathrm{also}$ used as the acceptor buffer in the SLM extraction on-disc. Sulphuric acid $\left(\mathrm{H}_{2} \mathrm{SO}_{4}\right)$ was used for the acidification of the donor. The samples for the characterization of the SLM extraction on-disc were prepared using control cell culture supernatant (control supernatant) obtained after 24 h $E$. coli culture, from CBJ786 strain (not pHCA producing). The $\mathrm{pH}$ of the Control supernatant was 5.8 at $24 \mathrm{~h}$ culture.

For the organic liquid membrane two different oils were tested, $n$-undecane, dihexyl ether (DHE) and DHE with trioctyl phosphine oxid (TOPO). Aqueous solutions were made with ultrapure water obtained from a Milli-Q® purification system (Millipore Corporation, Billerica, MA, USA). All the chemicals were purchased from SigmaAldrich® Co. (St. Louis, MO, USA), unless otherwise stated.

E. coli culture. pHCA producing E. coli strain was modified as described by Jendresen et al. $^{46}$, from the TAL expression strain BL21(DE3)pLysS (Invitrogen/Life Technologies), plasmids encoding the tyrosine ammonia-lyase FjTAL from Flavobacterium johnsoniae (CBJ800). Both the control strain (CBJ786) and pHCA producing strain (CBJ800) were cultured in M9 medium containing $10 \mathrm{~g} / \mathrm{L}$ glucose, $2 \mathrm{mM}$ tyrosine and $1 \mathrm{mM}$ IPTG up to $24 \mathrm{~h}$ in $180 \mathrm{~mL} \mathrm{M} 9$ medium in $500 \mathrm{~mL}$-flasks in a temperature-controlled orbital shaker $\left(30^{\circ} \mathrm{C}, 250 \mathrm{rpm}\right)$. Samples were taken at 6, 9, and $24 \mathrm{~h}$, and the supernatant was obtained by double centrifugation (10 $\mathrm{min}$ at $10000 \mathrm{~g}, 4{ }^{\circ} \mathrm{C}$ ) and filtration through a $0.2 \mu \mathrm{m}$ filter.

HPLC analysis. pHCA detection was performed using a Waltham HPLC system (Thermo Scientific, MA, USA) equipped with a UV detector (333 nm) as previously described (Jendresen et al., 2015), using a $5 \mu \mathrm{L}$ injection volume. The separation was achieved in a Discovery HS F5 column ( $3 \mu \mathrm{m}$ particle size, $15 \mathrm{~cm} \times 4.6 \mathrm{~mm})$ at $30^{\circ} \mathrm{C}$ under gradient elution with a two-solvent system (A: $10 \mathrm{mM}$ ammonium formate ( $\mathrm{pH} 3.0$ adjusted with formic acid) and B: acetonitrile) at a flow rate of $0.7 \mathrm{~mL} / \mathrm{min}$. The elution started at $5 \% \mathrm{~B}$ followed by a linear increase of the fraction to $60 \%$ between 1.5 and $7 \mathrm{~min}$ after injection. The fraction of B was decreased back to $5 \%$ between 9.5 and $9.6 \mathrm{~min}$, remaining there until $12 \mathrm{~min}$.

Electrode fabrication. Electrodes were fabricated on poly methyl methacrylate (PMMA Goodfellow Cambridge Limited, England) substrates using stencil-lithography and electron beam evaporation to deposit $200 \mathrm{~nm}$ gold on an adhesion layer of $20 \mathrm{~nm}$ chromium. The stencillithography method used here has previously been described in detail by Sanger et al. ${ }^{15}$ The area of the exposed working electrode is $0.69 \mathrm{~mm}^{2}$.

Design, fabrication, and assembly of the LoD. In order to facilitate the on-disc SLM extraction and detection, the microfluidic system was designed to have a donor unit and a combined acceptor and detection unit (Figure 1a). The electrodes placed in the bottom of the acceptor-detection units were connected to a potentiostat through springloaded pins, fixed to a custom-made printed circuit board (PCB) (Figure 1a). The $230 \mu \mathrm{L}$ volume sample was loaded into the donor unit through the inlet holes (Figure 1b). The acceptor unit $(3 \mu \mathrm{L})$ is connected to the donor unit through the microchannel, but separated by the nanoporous SLM (Figure 1a,b). The volume of the donor unit was designed to be considerable larger than the acceptor, to enable 
efficient long term extraction and enrichment during rotation. The addition of the acceptor buffer was achieved by placing two openings, acceptor in/out, at both side of the chamber, as shown in Figure $1 \mathrm{~b}$. The impregnation of the nanoporous membrane with the organic solvent was performed through an inlet placed close to the waste chamber (Figure 1b). The bottom of the acceptor-detection unit was formed by the PMMA substrate with eight embedded electrode arrays (working, counter and reference electrode (WE, CE and RE)) (Figure 1c).

The microfluidic system $(\varnothing 10 \mathrm{~cm})$ was designed using CAD software (AutoCAD, Autodesk, USA), and fabricated from 0.25 to $2 \mathrm{~mm}$ thick, optically clear, PMMA using a laser cutter (Epilog Mini 18, 30 W, from Epilog, USA). Except for the bottom substrate with electrodes, the entire microfluidic disc, including both donor and acceptor units, were assembled using thermal bonding. The bonding was carried out using a hydraulic press (PW20, Paul-Otto Weber $\mathrm{GmbH}$, Remshalden, Germany) at $85^{\circ} \mathrm{C}, 25$ bar pressure for 40 minutes, after activating the PMMA layers with isopropanol and 2 minutes UV treatment. The nanoporous polypropylene (PP) membrane (Celgard 2500, Celgard, LLC, Charlotte, NC, USA) with 55\% porosity, 64 $\mathrm{nm}$ pore size, and $25 \mu \mathrm{m}$ thickness, cut in $4 \times 6 \mathrm{~mm}$ pieces, was sandwiched between the donor and acceptor unit during bonding. The precise assembly of the PP membrane between the donor and acceptor unit was achieved by fixing the nanoporous membrane in place with a silicon tape $(1 \times 1 \mathrm{~mm}, 10 \mu \mathrm{m}$ thickness, INT TA106, Intertronics, Oxfordshire, UK). The detection unit, with incorporated electrodes, was created by placing a $150 \mu \mathrm{m}$ thick, pressure sensitive adhesive (PSA) tape (ARcare 90106, Adhesive Research, Ireland) between the bottom of the acceptor unit and the PMMA layers with the electrodes. The PSA layer was cut using a generic table top vinyl cutter (Silhouette Studio V3, Silhouette, USA). The whole assembled device was $5.5 \mathrm{~mm}$ thick, composed of a 3.25 $\mathrm{mm}$ donor unit and $2.25 \mathrm{~mm}$ thick acceptor-detection unit (with electrodes). a

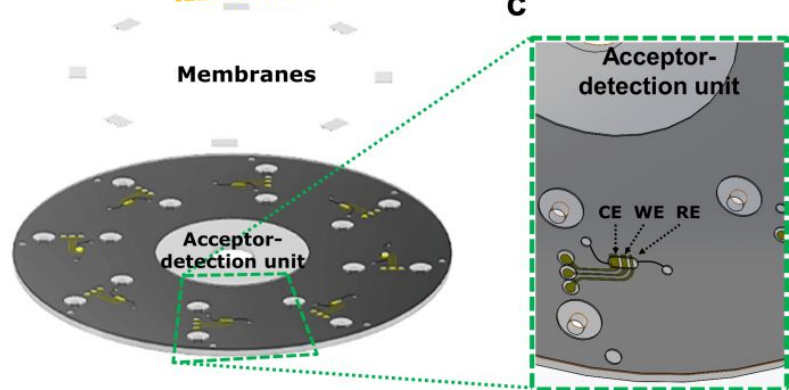

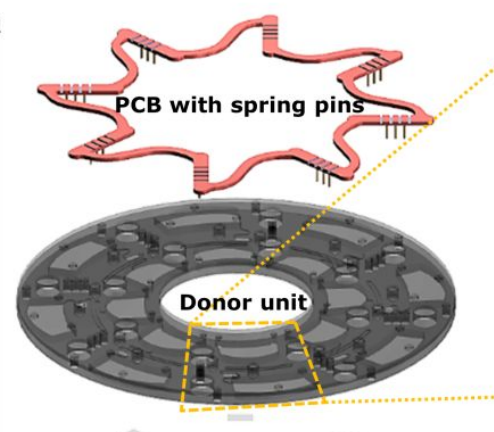

b

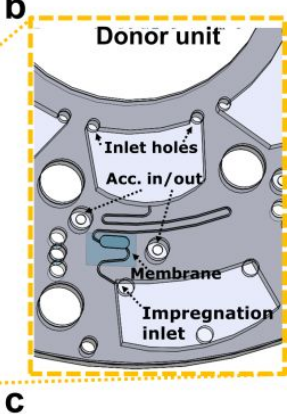

Figure 1: (a) Exploded view of the LoD device with integrated SLM extraction and embedded electrodes for detection. All dark grey parts are poly methyl methacrylate (PMMA). (b) Schematic of the donor unit, and (c) acceptor-detection unit with electrodes, labeled as a counter electrode (CE), a working electrode (WE) and a reference electrode (RE).

Working principle of the SLM extraction and electrochemical detection on the disc. The SLM extraction unit is composed of 3 parts: (1) An organic phase or oil, supported by a nanoporous polymer membrane, the SLM, is sandwiched between two aqueous phases, (2) a donor ( $\mathrm{pH} \mathrm{2)}$ and (3) an acceptor (pH 7.6) (Figure 2a,b). The donor, containing the supernatant, is first acidified with $\mathrm{H}_{2} \mathrm{SO}_{4}$. The donor and acceptor buffers, as well as the organic phase, were introduced in the designated opening of the LoD device (Figure 1a and 2a). By adjusting the $\mathrm{pH}$ of the sample/donor solution to $\mathrm{pH} 2$, which is below the $\mathrm{p} K_{a}$ of the carboxylic group of pHCA $(\mathrm{pKa}=4.34),{ }^{53}$ the target analyte, pHCA becomes neutral. However, since the substrate, Tyr, has a basic amino-group ( $\mathrm{pKa}=9.11)$, the molecule remains positively charged at $\mathrm{pH} \mathrm{2}$, as schematically represented in Figure $2 \mathrm{~b}$. The neutral pHCA diffuses through the organic SLM while the charged Tyr remains in the donor phase.
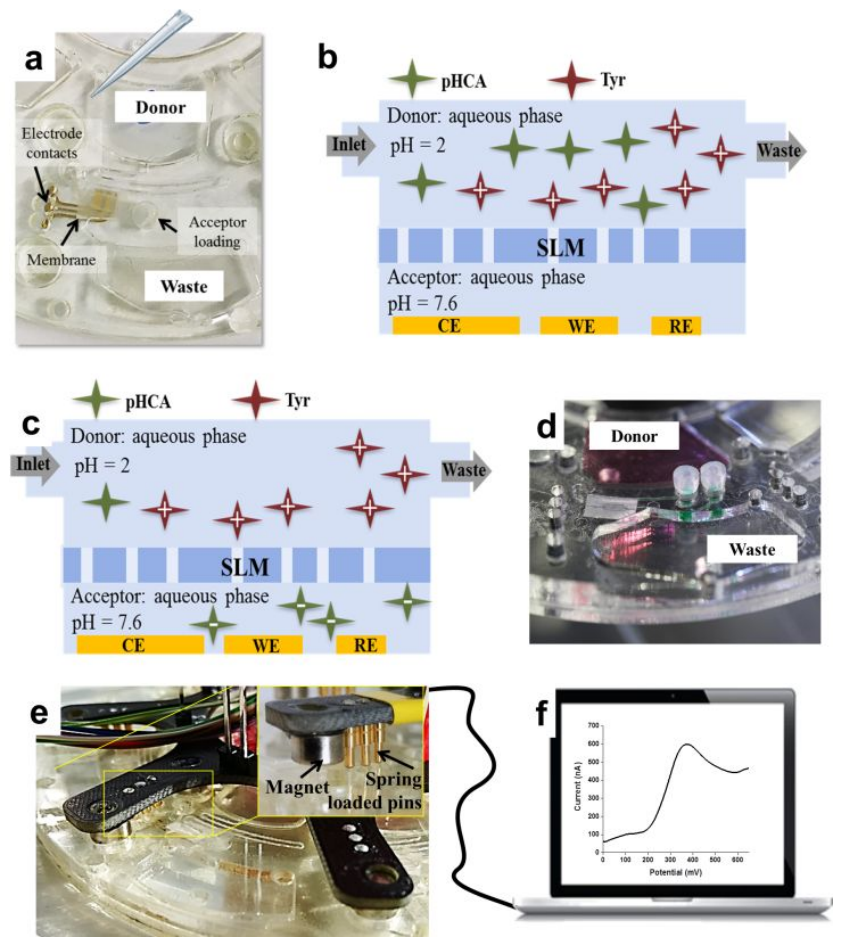

Figure 2: (a) Photograph, top view, of the SLM extractiondetection unit and representation of the loading of the acidified sample in the donor load chamber. (b) Schematic representation of the processes in the acceptor unit, pHCA becomes neutral, while Tyr is positively charged. (c) Representation of the extraction process, neutral pHCA diffuse to the SLM to the acceptor site, where becomes negatively charged. (d) Static image of the SLM extraction unit during the centrifugation process. (e) Photograph of the custom-made PCB with incorporated spring loaded pins, which facilitates the connection of the electrodes to 
the potentiostat. (f) Representation of a typical electrochemical signal a square wave voltammetry (SWV).

pHCA reaches the acceptor phase by simple mass diffusion through the organic SLM (Figure 2c). By keeping the acceptor buffer at $\mathrm{pH} 7.6$, which is well above the $\mathrm{p} K_{a}$ of pHCA, the molecule becomes deprotonated/charged, and unable to diffuse back into the organic SLM (Figure 2c). To facilitate the enrichment of pHCA in the acceptor phase, the extraction on the LoD was performed during rotation, using a spin-stand (Figure 2d). During rotation, a flow is induced (Video, V1) that enables control of the contact time between donor and SLM membrane, where the pHCA extraction takes place. The spin stand consists of an electronic triggering system for the high-resolution camera (pco.pixelfly, PCO AG, Kelheim, Germany), similar to the one described by Grumann et. al, $^{54}$ and a spinning motor (Maxon motor, model RE 35) controlled by custom-made software in a LabVIEW environment. After extraction, when the total donor volume had been transferred to the acceptor side, the system was left for 10 minutes to equilibrate, followed by electrochemical detection of pHCA (Figure 2e,f), as described later in the 'Electrochemical detection' section. The stable contact between the LoD device and the PCB was achieved as described ${ }^{55}$ using rod magnets of $\emptyset 4 \mathrm{~mm}$ and $3 \mathrm{~mm}$ height (Webcraft $\mathrm{GmbH}$, Gottmadingen, Germany) incorporated in the PCB and in the bottom PMMA layer (Figure 2e).

Electrochemical detection. All electrochemical measurements were performed at room temperature with an eight-channel potentiostat $\mathrm{CHI} 1030 \mathrm{~A}$ potentiostat $(\mathrm{CH}$ Instruments (CHI), Inc., Austin, TX, USA), and potentials were applied vs. on-disc Au pseudo-RE. The electrodes were characterized with cyclic voltammetry (CV) in a potential range from $-400 \mathrm{mV}$ to $400 \mathrm{mV}$ at $50 \mathrm{mV} / \mathrm{sec}$ scan rate, in $10 \mathrm{mM}$ ferri/ferrocyanide, using PBS, $\mathrm{pH}$ 7.4, as supporting electrolyte. Square wave voltammetry (SWV), a sensitive and reliable electrochemical technique, ${ }^{56}$ was used for the detection of pHCA, at a potential range from 0 to $650 \mathrm{mV}$ in Tris buffer (pH 7.6) and in supernatant (from both producing and non pHCA producing strains) ( $\mathrm{pH}$ 5.8) in a potential range between 0 to $800 \mathrm{mV}$, with a step potential of $0.001 \mathrm{~V}$, a frequency of $10 \mathrm{~Hz}$ and an amplitude of $0.05 \mathrm{~V}$. Detection of Tyr was carried out using the same SWV parameters in the potential range from 0 to $800 \mathrm{mV}$ in both Tris buffer at pH 7.6 and in the control supernatant at $\mathrm{pH}$ 5.8. The electrodes were interfaced with the potentiostat through a PCB (Figure 2e) and measurements were performed under static conditions. The electrochemical characterization, pre-calibration, as well as the measurement of the sample and controls before extraction, were performed under static condition using the on-disc electrodes in an 'open vial system' (Figure S2b) with volume of $40 \mu \mathrm{L}$. The linear calibration curve was further used for calculating the quantity of pHCA in real samples.

Evaluation of the extraction time on the centrifugal platform. The visualization and monitoring of the on-disc extraction process during rotation, was carried out by capturing images with a high-speed camera and stroboscopic light synchronized with the spinning of the disc. From the obtained images we were able to calculate the extraction time.

Data analysis. For the calibration curves, peak heights were obtained from the original data files using the CHI data analysis software and plotted against the corresponding concentration. The data plots were prepared using OriginPro 2015 (OriginLab Corporation Northampton, MA, USA). The presented SWVs are presented as recorded or background-subtracted and baseline-corrected to improve the visualization and identification of peaks over the baseline without introducing any artifacts. The enrichment factor (EF) was calculated by dividing the donor concentration before, with the acceptor concentration after SLM extraction.

\section{Results and Discussion}

Optimization of the SLM extraction. SLM extraction enables efficient sample clean-up, extraction and enrichment of target analytes. To enhance extraction efficiency and selectivity, the organic liquid used in the SLM can be modified with complexation agents, ${ }^{29,31}$ or additives to change the polarity of the liquid..$^{57,58}$ The extraction can also be coupled with affinity-based capturing of the target analyte through immuno-affinity interactions in the acceptor. ${ }^{15}$ However, for the extraction and trapping of organic acids or bases, the most commonly used approach is to use the $\mathrm{pH}$ differences between donor and acceptor, ${ }^{59}$ which is applicable for the extraction of our target pHCA. Accordingly, experiments were carried out to find a suitable organic liquid for trapping of this analyte. Three different organic liquids were selected based on their increasing polarity, $n$-undecane, DHE, and DHE with $5 \% \mathrm{TOPO}^{59}$, undecane being the most apolar, and DHE with TOPO the most polar. Their ability to selectively extract pHCA in presence of Tyr was evaluated using a static SLM extraction chip (Figure S1a). The content of the acceptor, after SLM extraction, was electrochemically evaluated with SWV, as described in supporting information and presented on Figure S1. We found that while detecting pHCA in presence of Tyr is problematic (Figure S1b), pHCA after SLM extraction can be clearly detected, since it gets efficiently separated from Tyr and extracted to the acceptor phase (Figure S1c). When comparing the tested organic solvents we found that the highest EF was obtained with the more polar oils, DHE, and DHE with $5 \%$ TOPO (Figure S1d). Since adding TOPO to the liquid membrane complicates the process, and since no discernible gain was seen from adding it, pure DHE was chosen as the liquid membrane for all further experiments.

Reproducibility of on-disc electrode arrays fabricated on a plastic support. For integration in the microfluidic device, electrodes can be fabricated on silicon ${ }^{37}$, glass ${ }^{36}$, plastic $^{60,61}$ or paper ${ }^{62}$ using either complex and timeconsuming lithographic approaches, ${ }^{36,37,60}$ or by faster and less expensive methods based on screen printing, ${ }^{61,62}$ or ebeam evaporation using a stencil or mask, ${ }^{15,63,64}$. The 8 electrode array, fabricated by e-beam evaporation on PMMA support and integrated into the LoD device, showed a high degree of reproducibility, as indicated from the eight overlaying CVs in Figure 3. 


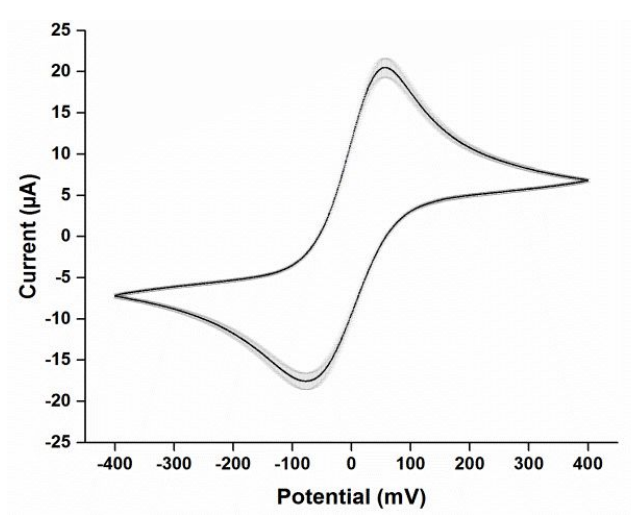

Figure 3: Overlay of eight cyclic voltammograms (CV) from 8 separate electrodes on the same disc, obtained at a scan-rate of $50 \mathrm{mV} / \mathrm{sec}$ and $10 \mathrm{mM}$ ferri/ferrocyanide in PBS, $\mathrm{pH} 7.4$, to illustrate the performance and reproducibility of the fabricated electrodes (potential vs. Au pseudo-reference electrode (RE)).

As presented in Figure 3, the differences between oxidation and reduction peak currents in the CVs recorded from eight electrodes in the LoD array was $+20.4 \pm 1.4 \mu \mathrm{A}$ and $-17.7 \pm 1.0 \mu \mathrm{A}$ respectively. The peak potential separation $(133 \pm 5 \mathrm{mV}$ ) was comparable with data obtained with gold electrodes fabricated on glass ${ }^{36}$, plastic $^{60}$ or silicon substrates ${ }^{37}$.

Electrochemical detection of pHCA. The sensors were pre-calibrated and calibration curves (Figure 4) were obtained when plotting the peak height from SWVs. The relative standard variation was found to be below $15 \%$. The SWVs were recorded for various concentration of pHCA from 250 up to $1500 \mu \mathrm{M}$ in Tris buffer, pH 7.6 (acceptor buffer in the SLM extraction).

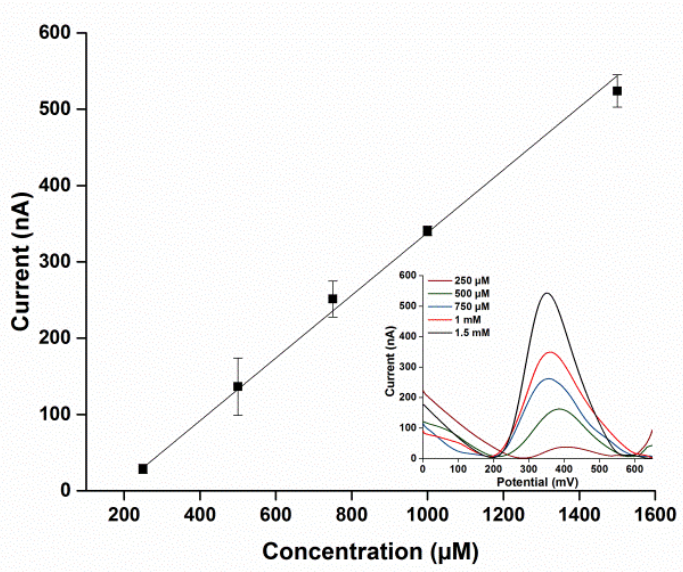

Figure 4: Calibration curve showing the correlation between peak current amplitudes from square wave voltammograms (SWVs) at different pHCA concentrations in Tris buffer, $\mathrm{pH}$ 7.6. Inset shows the SWVs of increasing pHCA concentration. The standard deviation was calculated from triplicates.

Direct electrochemical detection of pHCA and Tyr in a mixture is challenging given the proximity of their oxidation potentials (Figure S1b, Figure S2). ${ }^{38-40}$
Electrochemical detection of pHCA at the end of the bioprocess when Tyr is in low concentration (Figure S3) is feasible, ${ }^{15}$ however almost impossible when aiming at the quantification of pHCA during the entire production process because of the interference from Tyr (Figure S2). Implementing the SLM extraction step prior to electrochemical detection proved to effectively eliminate Tyr and solve this problem (Figure S1c). The developed extraction protocols were implemented on the LoD system as described in the Experimental section.

Figure 5 shows that the signal after extraction (dotted red line) is visibly different from the signal recorded before SLM extraction (red line). There is one clear peak at 380 $\mathrm{mV}$ attributed to pHCA in the SWV after extraction (dotted red line). This peak was not detectable in the SWV from $250 \mu \mathrm{M} \mathrm{pHCA}$ and $750 \mu \mathrm{M}$ Tyr spiked into the control supernatant ( $\mathrm{pH}$ 5.8) before extraction (red line). No other electrochemically active compound except pHCA was observed on the acceptor side (dotted red line). The intensity of the signal after extraction (dotted red line) is comparable with the signal recorded from $1 \mathrm{mM}$ pHCA in the acceptor buffer (green line), indicating that the pHCA was enriched at least 4 times after 40 min SLM extraction. As it is shown in Figure 5, there is a clear distinction between the signal recorded from Tyr (blue line) and SWV after SLM extraction (dotted red line), proving that Tyr is not present in the extract. The difference in oxidation potential of Tyr at pH 7.6 (blue line) and pH 5.8 (red line) is probably due to the $\mathrm{pH}$ dependent oxidation of Tyr. ${ }^{65} \mathrm{In}$ addition, the efficiency of the SLM extraction in separating pHCA from Tyr was further confirmed by HPLC (Figure S4).

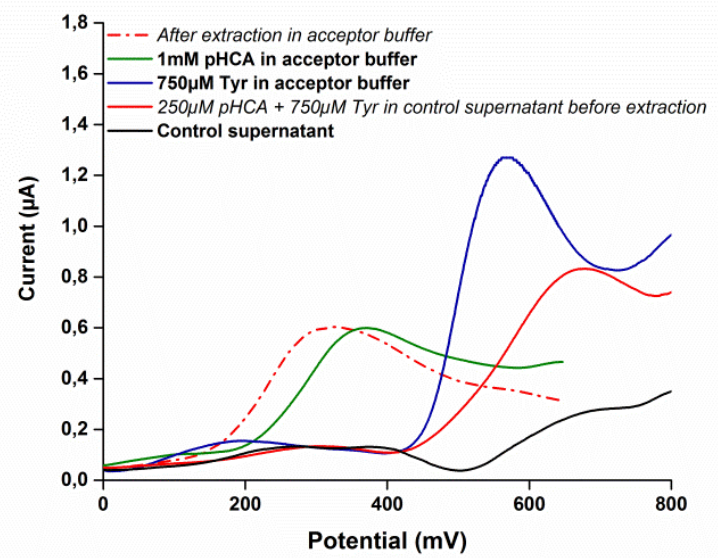

Figure 5: Representative SWVs showing the efficiency of the on-disc extraction. Signal recorded from $250 \mu \mathrm{M}$ pHCA $+750 \mu \mathrm{M}$ Tyr in the control supernatant $(\mathrm{pH} 5,8)$ before extraction (red line) and signal obtained in acceptor (Tris buffer) pH 7.6 after 40 min SLM extraction (dotted red line). SWVs of control supernatant (pH 5,8) in absence of pHCA and Tyr (black line), $1 \mathrm{mM}$ pHCA (green line) and $750 \mu \mathrm{M}$ Tyr (blue line) in acceptor buffer (pH 7.6) Potential vs. the on-disc Au pseudo-RE.

The peak potential difference between the signal recorded from $1 \mathrm{mM} \mathrm{pHCA}$ at $\mathrm{pH} 7.6$ in the acceptor and from 250 $\mu \mathrm{M} \mathrm{pHCA}$, extracted and enriched from the donor was $\sim 30$ 
$\mathrm{mV}$ (Figure 5). This is most likely due to the slight $\mathrm{pH}$ difference that can occur on the acceptor side through contamination from the donor side, due to ionic transfer. Similar behavior was noticed when pHCA was extracted from acidified PBS during optimization of the SLM extraction (Figure S1a,b). The pH-dependent electrochemical oxidation of pHCA is known $^{38}$ and also reported in our previous work. ${ }^{55}$

Characterization of SLM extraction on the developed LoD device. The on-disc SLM extraction was characterized in terms of the EF as a function of the total extraction time. To mimic the case where pHCA is present in lower concentration than Tyr, $250 \mu \mathrm{M}$ pHCA and $750 \mu \mathrm{M}$ Tyr were spiked into the control supernatant that had been collected after $24 \mathrm{~h}$ bacterial culture.

Various extraction times from 10 to $45 \mathrm{~min}$ were evaluated, to find the maximum EF to be used when extracting from real samples. As observed in Figure 6, a clear increase in EF was observed until $\sim 40$ minut extraction time, after which a steady state is approached. The maximum EF in the on-disc extraction was found to be close to 4.7. Additionally, we found (Figure 6, inset) that under rotation ( 40 min extraction time) the EF was 5 times higher, than the EF obtained when the extraction was carried out for $40 \mathrm{~min}$ under static condition without flow.

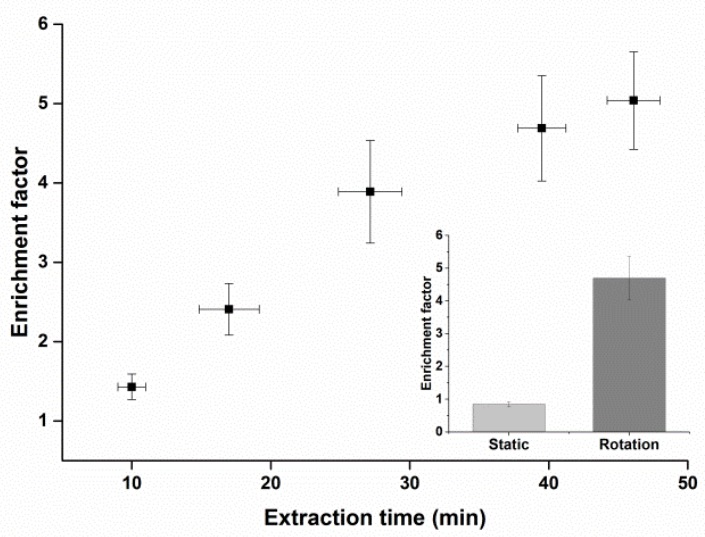

Figure 6: The enrichment obtained on-disc as function of extraction time. The standard deviation was calculated based on three repetitions. The enrichment under static condition and during extraction under rotation both cases with 40 min extraction time (inset).

By knowing the correlation between extraction time and the on-disc enrichment we can back calculate and quantify the original concentration of pHCA in unknown samples.

Quantification of pHCA from bacterial supernatant and validation with HPLC. Bacterial supernatant samples of strain CBJ800 were taken at different time points $(6,9$ and $24 \mathrm{~h}$ ) during the pHCA production process. $230 \mu \mathrm{L}$ samples were placed in the donor chambers, acidified and extracted through the SLM into the acceptor chamber on the LoD platform. Electrochemical detection was performed directly after extraction using the electrochemical sensors integrated at the bottom of the acceptor chambers. Using the calibration curve and the correlation between extraction time and enrichment, the pHCA concentration in the samples could be calculated (Figure 6, EF vs. time).

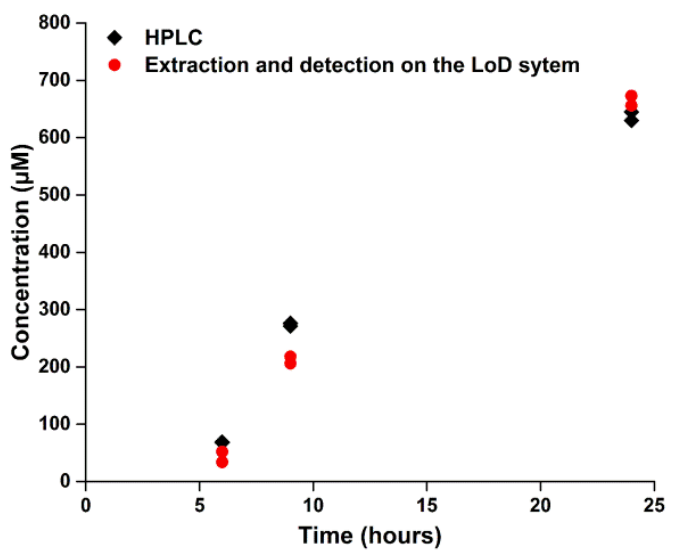

Figure 7: Quantification of pHCA concentration in samples from cell culture supernatant, obtained at 6, 9 and 24 hours, using the developed LoD device (red dots) and HPLC (black dots) $n=2$.

Figure 7 shows the calculated pHCA concentration in the samples measured electrochemically after the SLM extraction, as well as the concentration measured with HPLC. As can be seen, the data from the samples analyzed with HPLC were in good agreement with the data obtained from the on-disc SLM extraction combined with electrochemical detection.

\section{Conclusion}

The LoD system, integrating eight combined SLM extraction and electrochemical detection units, offers a time and cost effective, as well as an environmentally friendly, alternative for quantification of the bacterial metabolite, pHCA. It is a significant development compared to our previous work, ${ }^{15}$ since this device facilitates detection not only at the end of the production process but already at an earlier (6h) time point where the concentration of the target analyte (pHCA) is relatively low, typically below $100 \mu \mathrm{M}$ in the presence of high concentration of the main interfering compound (Tyr). The integrated SLM extraction enables not only the separation of pHCA from Tyrbut also facilitates sample enrichment. The developed platform is aimed at being single use due to its low production cost, however the electrodes can, when needed be reused. ${ }^{15}$ We have shown how this LoD SLM extraction/electrochemical platform can be used in "sample-to-answer" applications. Considering the speed of analysis, parallelization of the assay, and potential future multiplexing, the presented work demonstrates the advantages and potential of integrated sample pretreatment and detection for at-line bioprocess monitoring.

\section{Acknowledgments}

This work was financially supported by the European Research Council under the European Union's Seventh Framework Programme (FP7/2007-2013) grant no. 
320535-HERMES. The authors also acknowledge the support from the Novo Nordisk Foundation (grant no. NNF150C0015246) and from the IDUN project (grant no. DNRF122) funded by the Danish National Research Foundation and the Velux Foundations. The authors acknowledge the contribution of Senior lecturer Margareta Sandahl in the initial phase of setting up the SLM extraction and Laura Serioli for her help with part of CAD based illustration.

\section{ASSOCIATED CONTENT}

Supporting Information. Video showing the functionality of the fluidics. Initial optimization of the SLM extraction including electrochemical detection of pHCA in the presence and absence of tyrosine (Tyr) is control supernatant and the photograph of the LoD system for static measurement. E. coli growth curve and production rate of pHCA and consumption of Tyr during $24 \mathrm{~h}$ of culture. Chromatograms obtained from samples before and after SLM extraction. This material is available free of charge via the Internet at http://pubs.acs.org."

\section{AUTHOR INFORMATION}

\section{Corresponding Author}

*Corresponding authors: Kinga Zór, Technical University of Denmark, Department of Micro-and Nanotechnology, Ørsteds Plads, Building 345C, room 101E, $2800 \mathrm{Kgs}$. Lyngby, Denmark, Tel:+4545255751, email:kinzo@nanotech.dtu.dk

Sune Zoëga Andreasen, Technical University of Denmark, Department of Micro-and Nanotechnology, Produktionstorvet, Building: 423, room 124, $2800 \mathrm{Kgs}$. Lyngby, Denmark, Tel: +45258126, email: sunez@nanotech.dtu.dk

\section{Author Contributions}

+ These authors contributed equally to the work.

\section{REFERENCES}

(1) Manz, A.; Graber, N.; Widmer, H. áM., Miniaturized total chemical analysis systems: a novel concept for chemical sensing, Sensors Actuators B Chem. 1990, 1 (1), 244-248.

(2) Verpoorte, E.; Manz, A.; Lüdi, H.; Bruno, A. E.; Maystre, F.; Krattiger, B.; Widmer, H. M.; van der Schoot, B. H.; de Rooij, N. F., A silicon flow cell for optical detection in miniaturized total chemical analysis systems, Sensors Actuators B Chem. 1992, 6 (1-3), 66-70.

(3) Whitesides, G. M., The origins and the future of microfluidics, Nature 2006, 442 (7101), 368-373.

(4) Mark, D.; Haeberle, S.; Roth, G.; von Stetten, F.; Zengerle, R., Microfluidic lab-on-a-chip platforms: requirements, characteristics and applications, Chem. Soc. Rev. 2010, 39 (3), 1153-1182.

(5) Castillo-León, J.; Svendsen, W. E., Lab-on-a-Chip Devices and Micro-Total Analysis Systems, Springer International Publishing, Cham, 2015.

(6) Whitesides, G., Solving problems, Lab Chip 2010, 10 (18), 2317-2318.

(7) Mohammed, M. I.; Haswell, S.; Gibson, I., Lab-on-a-chip or Chip-in-a-lab: Challenges of Commercialization Lost in Translation, Procedia Technol. 2015, 20 (July), 54-59.

(8) Volpatti, L. R.; Yetisen, A. K., Commercialization of microfluidic devices, Trends Biotechnol. 2014, 32, 347-350.
(9) de Mello, A. J.; Beard, N., Dealing with real samples: sample pre-treatment in microfluidic systems, Lab Chip 2003, 3 (1), $11 \mathrm{~N}-19 \mathrm{~N}$.

(10) Giordano, B. C.; Burgi, D. S.; Hart, S. J.; Terray, A., On-line sample pre-concentration in microfluidic devices: A review, Anal. Chim. Acta 2012, 718, 11-24.

(11) Lee, B. S.; Lee, J.-N.; Park, J.-M.; Lee, J.-G.; Kim, S.; Cho, Y.-K.; Ko, C., A fully automated immunoassay from whole blood on a disc, Lab Chip 2009, 9 (11), 1548-1555.

(12) Kim, T.-H.; Abi-Samra, K.; Sunkara, V.; Park, D.-K.; Amasia, M.; Kim, N.; Kim, J.; Kim, H.; Madou, M.; Cho, Y.-K., Flowenhanced electrochemical immunosensors on centrifugal microfluidic platforms, Lab Chip 2013, 13 (18), 3747-3754.

(13) Zór, K.; Gáspár, S.; Hashimoto, M.; Suzuki, H.; Csöregi, E., High Temporal Resolution Monitoring of Fermentations Using an On-Line Amperometric Flow-Through Microdetector, Electroanalysis 2007, 19 (1), 43-48.

(14) Morelli, L.; Andreasen, S. Z.; Jendresen, C. B.; Nielsen, A. T.; Emneus, J.; Zor, K.; Boisen, A., Quantification of a bacterial secondary metabolite by SERS combined with SLM extraction for bioprocess monitoring, Analyst 2017, 142, 4553-4559.

(15) Sanger, K.; Zór, K.; Christian Bille Jendresen, A. H.; Amato, L.; Alex Toftgaard Nielsen, A. B., Lab-on-a-disc platform for screening of genetically modified E. coli cells via cell-free electrochemical detection of p-coumaric acid, Sensors Actuators B Chem. 2017, 253, 999-1005.

(16) Tudorache, M.; Zdrojewska, I. A.; Emnéus, J., Evaluation of progesterone content in saliva using magnetic particle-based immuno supported liquid membrane assay (m-ISLMA), Biosens. Bioelectron. 2006, 22 (2), 241-246.

(17) Tudorache, M.; Co, M.; Lifgren, H.; Emnéus, J., Ultrasensitive magnetic particle-based immunosupported liquid membrane assay, Anal. Chem. 2005, 77 (22), 7156-7162. Madou, M.; Zoval, J.; Jia, G.; Kido, H.; Kim, J.; Kim, N., Lab on a Cd, Annu. Rev. Biomed. Eng. 2006, 8 (1), 601-628.

(19) Strohmeier, O.; Keller, M.; Schwemmer, F.; Zehnle, S.; Mark D.; von Stetten, F.; Zengerle, R.; Paust, N., Centrifugal microfluidic platforms: advanced unit operations and applications, Chem. Soc. Rev. 2015, 44, 6187-6229.

Gorkin, R.; Park, J.-M. J.; Siegrist, J.; Amasia, M.; Lee, B. S.; Park, J.-M. J.; Kim, J.; Kim, H.; Madou, M.; Cho, Y.-K., Centrifugal microfluidics for biomedical applications, $L a b$ Chip 2010, 10 (14), 1758-1773.

(21) Nwankire, C. E.; Czugala, M.; Burger, R.; Fraser, K. J.; Connell, T. M.; Glennon, T.; Onwuliri, B. E.; Nduaguibe, I. E.; Diamond, D.; Ducrée, J., A portable centrifugal analyser for liver function screening, Biosens. Bioelectron. 2014, 56, 352-358. Nwankire, C. E.; Donohoe, G. G.; Zhang, X.; Siegrist, J.; Somers, M.; Kurzbuch, D.; Monaghan, R.; Kitsara, M.; Burger, R.; Hearty, S.; Murrell, J.; Martin, C.; Rook, M.; Barrett, L.; Daniels, S.; Mcdonagh, C.; Kennedy, R. O.; Ducrée, J., At-line bioprocess monitoring by immunoassay with rotationally controlled serial siphoning and integrated supercritical angle fluorescence optics, Anal. Chim. Acta 2013, 781, 54-62.

(23) Noroozi, Z.; Kido, H.; Peytavi, R.; Nakajima-Sasaki, R.; Jasinskas, A.; Micic, M.; Felgner, P. L.; Madou, M., A multiplexed immunoassay system based upon reciprocating centrifugal microfluidics, J. Rev. Sci. Instrum. 2011, 82 (6).

(24) Tang, M.; Wang, G.; Kong, S. K.; Ho, H. P., A review of biomedical centrifugal microfluidic platforms, Micromachines 2016, 7 (2).

(25) Cai, Z.; Xiang, J.; Chen, H.; Wang, W., Membrane-based valves and inward-pumping system for centrifugal microfluidic platforms, Sensors Actuators, B Chem. 2016, 228, 251-258.

(26) Audunsson, G., Determination of low parts per billion levels of amines in urine by liquid membrane sample cleanup directly coupled to a gas-liquid chromatograph, Anal. Chem. 1988, 60 (13), 1340-1347.

(27) Shen, Y.; Strom, L.; Jonsson, J.-A.; Tyler, G., Low-molecular organic acids in the rhizosphere soil solution of beech forest (Fagus Sylvatica L.) Cambisols determined by ion chromatography using supported liquid membrane enrichment technique, Soil Biol. Biochem. 1996, 38 (9), 
1163-1169.

Lee, J.; Lee, H. K.; Rasmussen, K. E.; Pedersen-Bjergaard, S., Environmental and bioanalytical applications of hollow fiber membrane liquid-phase microextraction: A review, Anal. Chim. Acta 2008, 624 (2), 253-268.

(29) Gao, L.; Jönsson, J. Å., Determination of Melamine in Fresh Milk with Hollow Fiber Liquid Phase Microextraction Based on Ion-Pair Mechanism Combined with High Performance Liquid Chromatography, Anal. Lett. 2012, 45 (16), $2310-$ 2323.

(30) Pálmarsdóttir, S.; Thordarson, E.; Edholm, L. E.; Jönsson, J. Å.; Mathiasson, L., Miniaturized supported liquid membrane device for selective on-line enrichment of basic drugs in plasma combined with capillary zone electrophoresis, Anal. Chem. 1997, 69 (9), 1732-1737.

(31) Sandahl, M.; Mathiasson, L.; Jönsson, J. Å., On-line automated sample preparation for liquid chromatography using parallel supported liquid membrane extraction and microporous membrane liquid-liquid extraction, J. Chromatogr. A 2002, 975 (1), 211-217.

(32) Gencoglu, A.; Minerick, A. R., Electrochemical detection techniques in micro- and nanofluidic devices, Microfluid. Nanofluidics 2014, 17 (5), 781-807.

(33) Nyholm, L., Electrochemical techniques for lab-on-a-chip applications, Analyst 2005, 130 (5), 599.

(34) Xu, X.; Zhang, S.; Chen, H.; Kong, J., Integration of electrochemistry in micro-total analysis systems for biochemical assays: recent developments, Talanta 2009, 80 (1), 8-18.

(35) Patou, F.; AlZahra'a Alatraktchi, F.; Kjægaard, C.; Dimaki, M.; Madsen, J.; Svendsen, W., Evolvable Smartphone-Based Platforms for Point-of-Care In-Vitro Diagnostics Applications, Diagnostics 2016, 6 (3), 33.

(36) Andreasen, S. Z.; Kwasny, D.; Amato, L.; Brogger, A. L.; Bosco, F. G.; Andersen, K. B.; Svendsen, W. E.; Boisen, A., Integrating Electrochemical Detection with Centrifugal Microfluidics for Real-Time and Fully Automated Sample Testing, RSC Adv 2015, 5 (22), 17187-17193.

(37) Zór, K.; Heiskanen, A.; Caviglia, C.; Vergani, M.; Landini, E.; Shah, F.; Carminati, M.; Martínez-Serrano, A.; Moreno, T. R.; Kokaia, M.; Benayahu, D.; Keresztes, Z.; Papkovsky, D.; Wollenberger, U.; Svendsen, W. E.; Dimaki, M.; Ferrari, G.; Raiteri, R.; Sampietro, M.; Dufva, M.; Emnéus, J., A compact multifunctional microfluidic platform for exploring cellular dynamics in real-time using electrochemical detection, $R S C$ Adv. 2014, 4 (109), 63761-63771.

(38) Janeiro, P.; Novak, I.; Seruga, M.; Oliveira-Brett, A. M. Electroanalytical Oxidation of p-Coumaric Acid, Anal. Lett 2007, 40 (17), 3309-3321.

(39) Quintana, C.; Suárez, S.; Hernández, L., Nanostructures on gold electrodes for the development of an L-tyrosine electrochemical sensor based on host-guest supramolecular interactions, Sensors Actuators, B Chem. 2010, 149 (1), 129135.

(40) Xu, Q.; Wang, S.-F., Electrocatalytic Oxidation and Direct Determination of L-Tyrosine by Square Wave Voltammetry at Multi-wall Carbon Nanotubes Modified Glassy Carbon Electrodes, Microchim. Acta 2005, 151 (1-2), 47-52.

(41) Lindegård, B.; Bjork, H.; Jonsson, J. Å.; Mathiasson, L.; Olsson, A. M., Automated Column Liquid Chromatographic Determination of a Basic Drug in Blood Plasma Using the Supported Liquid Membrane Technique for Sample Pretreatment, Anal. Chem. 1994, 66 (24), 4490-4497.

(42) Jönsson, J. Å.; Mathiasson, L., Liquid membrane extraction in analytical sample preparation: I. Principles, TrAC Trends Anal. Chem. 1999, 18 (5), 318-325.

(43) Kadoma, Y.; Fujisawa, S., A Comparative Study of the Radicalscavenging Activity of the Phenolcarboxylic Acids Caffeic Acid, p-Coumaric Acid, Chlorogenic Acid and Ferulic Acid, With or Without 2-Mercaptoethanol, a Thiol, Using the Induction Period Method, Molecules 2008, 13 (10), 24882499.

(44) Balasundram, N.; Sundram, K.; Samman, S., Phenolic compounds in plants and agri-industrial by-products:
Antioxidant activity, occurrence, and potential uses, Food Chem. 2006, 99 (1), 191-203.

(45) Nishiyama, Y.; Yun, C. S.; Matsuda, F.; Sasaki, T.; Saito, K. Tozawa, Y., Expression of bacterial tyrosine ammonia-lyase creates a novel p-coumaric acid pathway in the biosynthesis of phenylpropanoids in Arabidopsis, Planta 2010, 232 (1), 209-218.

(46) Jendresen, C. B.; Stahlhut, S. G.; Li, M.; Gaspar, P.; Siedler, S.; Förster, J.; Maury, J.; Borodina, I.; Nielsen, A. T., Highly active and specific tyrosine ammonia-lyases from diverse origins enable enhanced production of aromatic compounds in bacteria and Saccharomyces cerevisiae, Appl. Environ. Microbiol. 2015, 81 (13), 4458-4476.

(47) Rodriguez, A.; Kildegaard, K. R.; Li, M.; Borodina, I.; Nielsen, J., Establishment of a yeast platform strain for production of p-coumaric acid through metabolic engineering of aromatic amino acid biosynthesis, Metab. Eng. 2015, 31, 181-188.

(48) Vannelli, T.; Wei Qi, W.; Sweigard, J.; Gatenby, A. a.; Sariaslani, F. S., Production of p-hydroxycinnamic acid from glucose in Saccharomyces cerevisiae and Escherichia coli by expression of heterologous genes from plants and fungi, Metab. Eng. 2007, 9 (2), 142-151.

(49) Glavnik, V.; Simonovska, B.; Vovk, I.; Mutavd, D.; Danijela, A. Babi, S., Quantification of (-) -Epicatechin and Procyanidin B2 in Chocolates, J Planar Chromat. 2011, 24, 482-486.

(50) Dietrich, J. A.; McKee, A. E.; Keasling, J. D., High-Throughput Metabolic Engineering: Advances in Small-Molecule Screening and Selection, Annu. Rev. Biochem. 2010, 79 (1), 563-590.

(51) Miller, J. H., Experiments in molecular genetics, Cold Spring Harb. Lab. Press. Cold Spring Harb. NY 1972.

(52) Morelli, L.; Andreasen, S. Z.; Jendresen, C. B.; Nielsen, A. T.; Emnéus, J.; Zór, K.; Boisen, A., Quantification of a bacterial secondary metabolite by SERS combined with SLM extraction for bioprocess monitoring, Analyst 2017, 142 (23), 4553-4559.

(53) Aguilar-Hernández, I.; Afseth, N. K.; López-Luke, T.; Contreras-Torres, F. F.; Wold, J. P.; Ornelas-Soto, N., Surface enhanced Raman spectroscopy of phenolic antioxidants: A systematic evaluation of ferulic acid, p-coumaric acid, caffeic acid and sinapic acid, Vib. Spectrosc. 2017, 89, 113-122.

(54) Grumann, M.; Brenner, T.; Beer, C.; Zengerle, R.; Ducrée, J., Visualization of flow patterning in high-speed centrifugal microfluidics, Rev. Sci. Instrum. 2005, 76 (2), 025101.

(55) Sanger, K.; Zór, K.; Bille Jendresen, C.; Heiskanen, A.; Amato, L.; Toftgaard Nielsen, A.; Boisen, A., Lab-on-a-disc platform for screening of genetically modified E. coli cells via cell-free electrochemical detection of p-Coumaric acid, Sensors Actuators B Chem. 2017, 253, 999-1005.

(56) Chen, A.; Shah, B., Electrochemical sensing and biosensing based on square wave voltammetry, Anal. Methods 2013, 5 (9), 2158.

(57) Shen, Y.; Grönberg, L.; Jönsson, J. Å., Experimental studies on the enrichment of carboxylic acids with tri-n-octylphosphine oxide as extractant in a supported liquid membrane, Anal. Chim. Acta 1994, 292 (1-2), 31-39.

(58) Jönsson, J. Å.; Mathiasson, L., Liquid membrane extraction in analytical sample preparation, TrAC Trends Anal. Chem. 1999, 18 (5), 325-334.

(59) Jönsson, J. A.; Mathiasson, L., Membrane extraction techniques in bioanalysis, Chromatographia 2000, 52 (S1), S8-S11.

(60) Matteucci, M.; Heiskanen, A.; Zór, K.; Emnéus, J.; Taboryski, R., Comparison of Ultrasonic Welding and Thermal Bonding for the Integration of Thin Film Metal Electrodes in Injection Molded Polymeric Lab-on-Chip Systems for Electrochemistry, Sensors 2016, 16 (12), 1795.

(61) Yoon, Y. J.; Li, K. H. H.; Low, Y. Z.; Yoon, J.; Ng, S. H., Microfluidics biosensor chip with integrated screen-printed electrodes for amperometric detection of nerve agent, Sensors Actuators, B Chem. 2014, 198, 233-238.

(62) Nie, Z.; Nijhuis, C. A.; Gong, J.; Chen, X.; Kumachev, A.; Martinez, A. W.; Narovlyansky, M.; Whitesides, G. M. Electrochemical sensing in paper-based microfluidic devices, 
Lab Chip 2010, 10 (4), 477-483.

(63) Xu, Y.; Huang, L.-B.; Yung, K.-L.; Xie, Y.-C.; Lee, T. M.-H., Low cost fabrication of microelectrodes on plastic substrate, Microsyst. Technol. 2011, 17 (3), 361-366.

(64) Amato, L.; Sanger, K.; Tehrani, S. E.; Burger, R.; Caviglia, C.; Andreasen, Z.; Heiskanen, A.; Emnéus, J.; Boisen, A., A labon-a-disc platform for trapping of cells, monitoring of cell behaviour and evaluation of redox metabolism, Proceedings of MicroTAS 2015; Chemical and Biological Microsystems Society, 2015; pp 1299-1302.

(65) Ivandini, T. A.; Sarada, B. V.; Terashima, C.; Rao, T. N.; Tryk, D. A.; Ishiguro, H.; Kubota, Y.; Fujishima, A., G radient liquid chromatography of leucine-enkephalin peptide and its metabolites with electrochemical detection using highly boron-doped diamond electrode, J. Chromatogr. B Anal. Technol. Biomed. Life Sci. 2003, 791 (1-2), 63-72. 
a
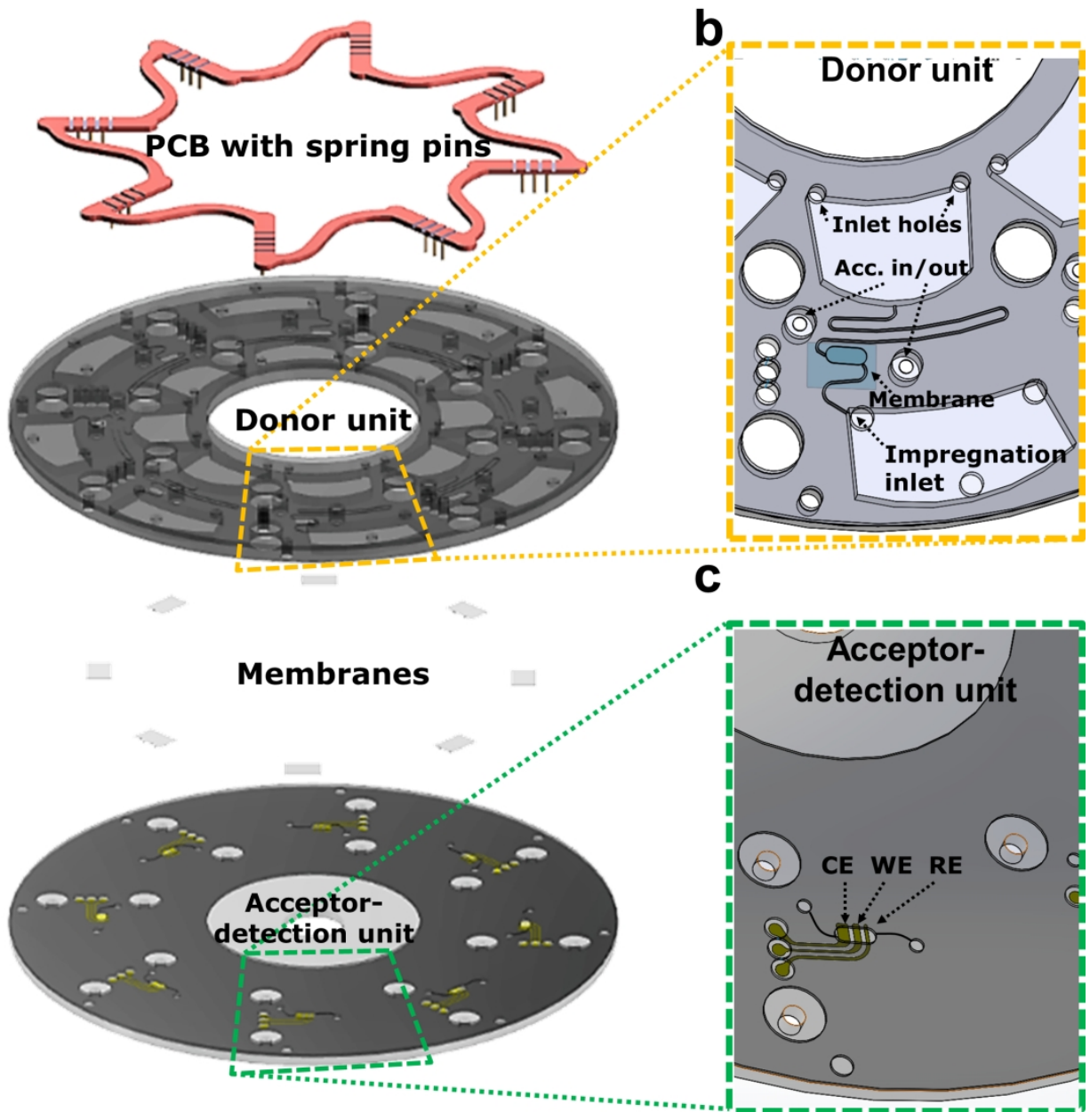

Figure 1: (a) Exploded view of the LoD device with integrated SLM extraction and embedded electrodes for detection. All dark grey parts are poly methyl methacrylate (PMMA). (b) Schematic of the donor unit, and (c) acceptor-detection unit with electrodes, labeled as a counter electrode (CE), a working electrode (WE) and a reference electrode (RE).

$$
191 \times 187 \mathrm{~mm}(300 \times 300 \text { DPI })
$$




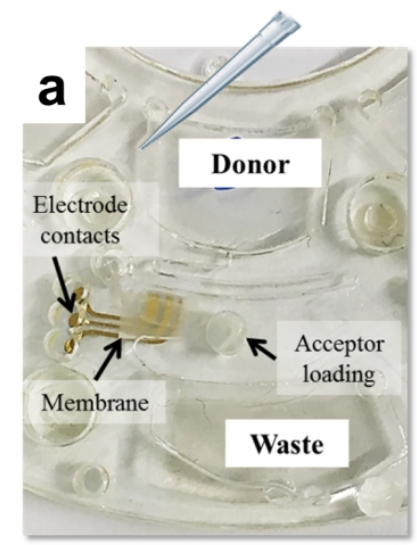

b
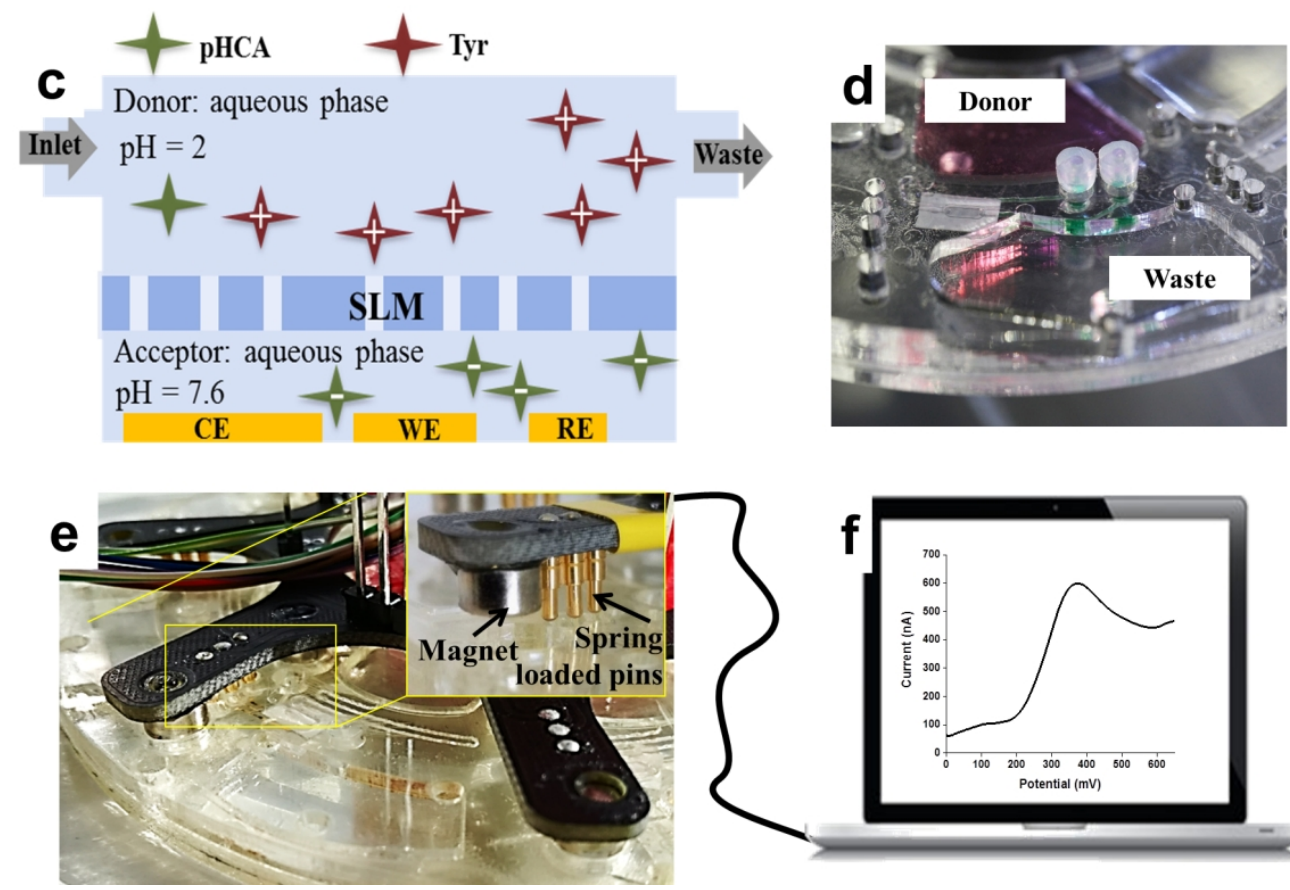

Acceptor: aqueous phase $\mathrm{pH}=7.6$

CE

Figure 2: (a) Photograph, top view, of the SLM extraction-detection unit and representation of the loading of the acidified sample in the donor load chamber. (b) Schematic representation of the processes in the acceptor unit, pHCA becomes neutral, while Tyr is positively charged. (c) Representation of the extraction process, neutral pHCA diffuse to the SLM to the acceptor site, where becomes negatively charged. (d) Static image of the SLM extraction unit during the centrifugation process. (e) Photograph of the custom-made PCB with incorporated spring loaded pins, which facilitates the connection of the electrodes to the potentiostat.

(f) Representation of a typical electrochemical signal a square wave voltammetry (SWV).

$150 \times 171 \mathrm{~mm}(300 \times 300 \mathrm{DPI})$ 
Figure 3: Overlay of eight cyclic voltammograms (CV) from the same disc, obtained at a scan-rate of

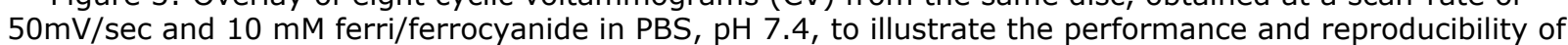
the fabricated electrodes (potential vs. Au pseudo-reference electrode (RE)).

$79 \times 61 \mathrm{~mm}(300 \times 300$ DPI $)$ 


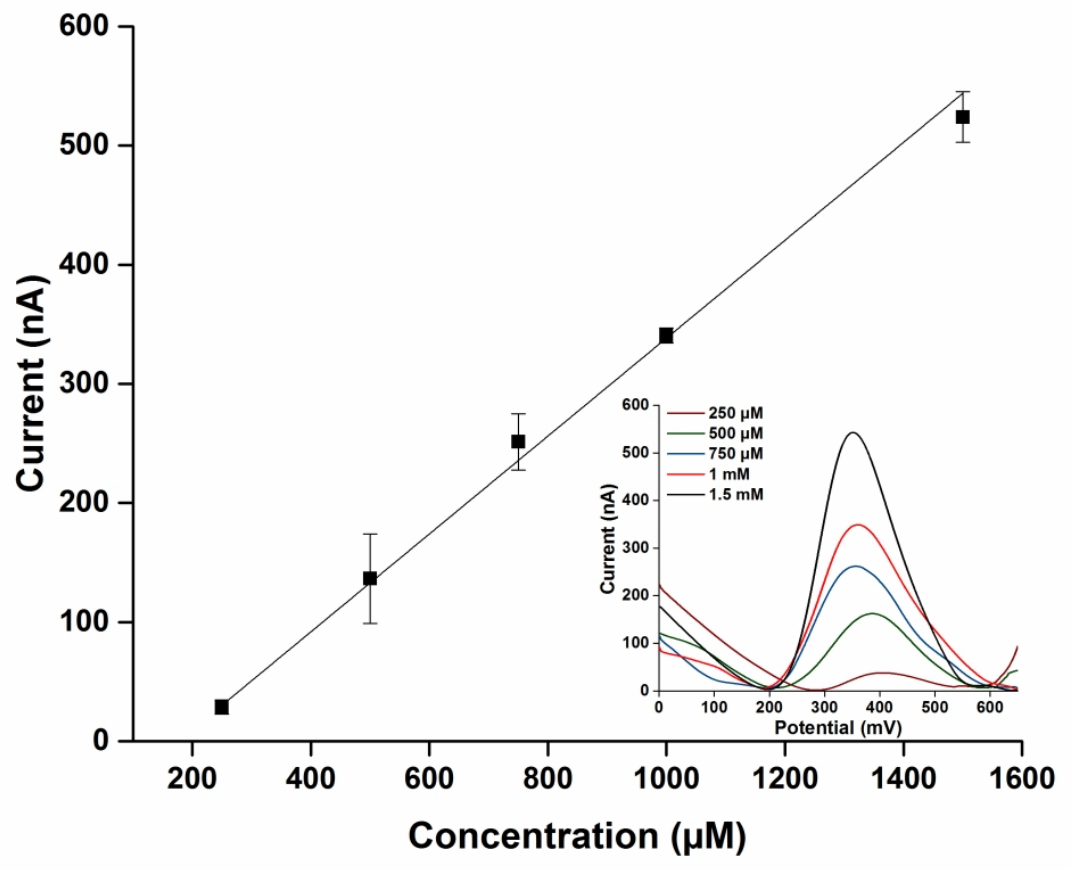

Figure 4: Calibration curve showing the correlation be-tween peak current amplitudes from square wave voltammograms (SWVs) at different pHCA concentrations in Tris buffer, $\mathrm{pH} 7.6$. Inset shows the SWVs of increasing pHCA concentration. The standard deviation was calculat-ed from triplicates.

$272 \times 208 \mathrm{~mm}(300 \times 300 \mathrm{DPI})$ 


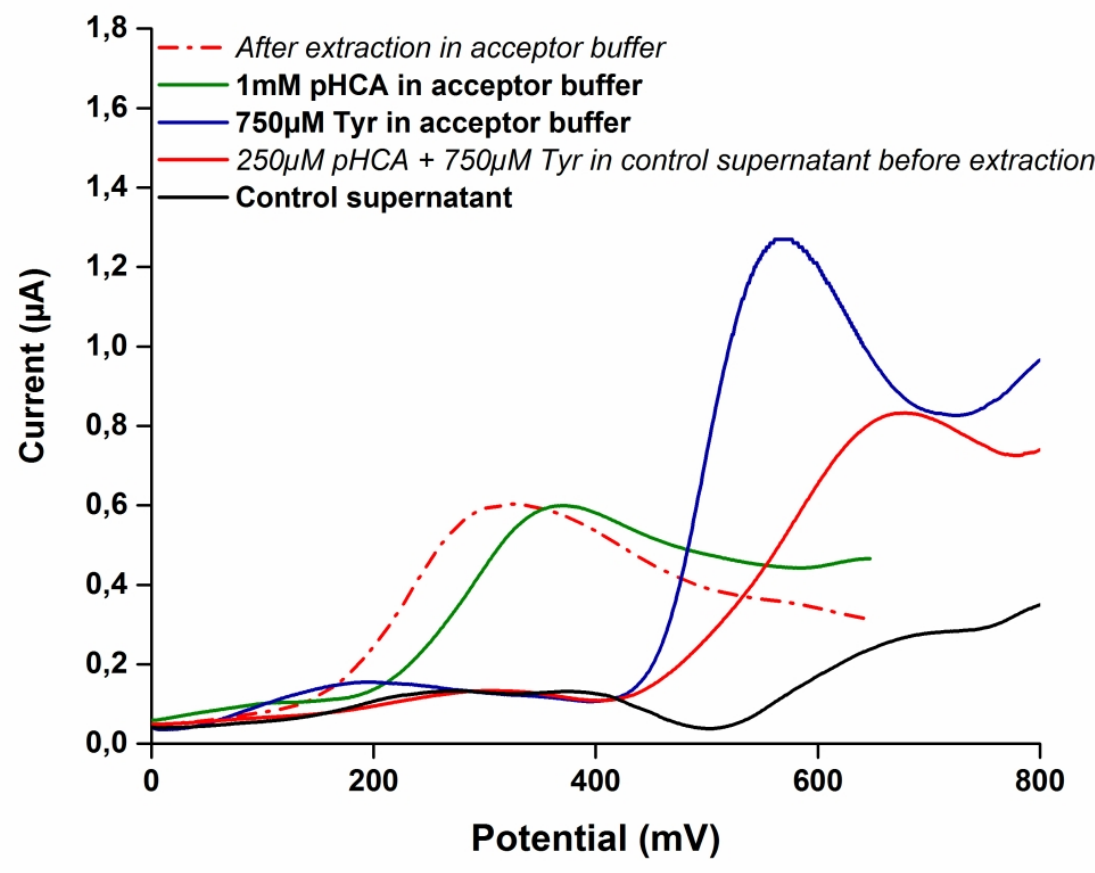

Figure 5: SWVs showing the efficiency of the on-disc extraction. Signal recorded from $250 \mu \mathrm{M} \mathrm{pHCA}+750$ $\mu \mathrm{M}$ Tyr in the control supernatant ( $\mathrm{pH} \mathrm{5,8)} \mathrm{before} \mathrm{extraction} \mathrm{(red} \mathrm{line)} \mathrm{and} \mathrm{signal} \mathrm{obtained} \mathrm{in} \mathrm{acceptor} \mathrm{(Tris}$ buffer) pH 7.6 after 40 min SLM extraction (dotted red line). SWVs of control supernatant $(\mathrm{pH} 5,8)$ in absence of pHCA and Tyr (black line), $1 \mathrm{mM}$ pHCA (green line) and $750 \mu \mathrm{M}$ Tyr (blue line) in acceptor buffer (pH 7.6) Potential vs. the on-disc Au pseudo-RE.

$272 \times 208 \mathrm{~mm}(300 \times 300$ DPI $)$ 


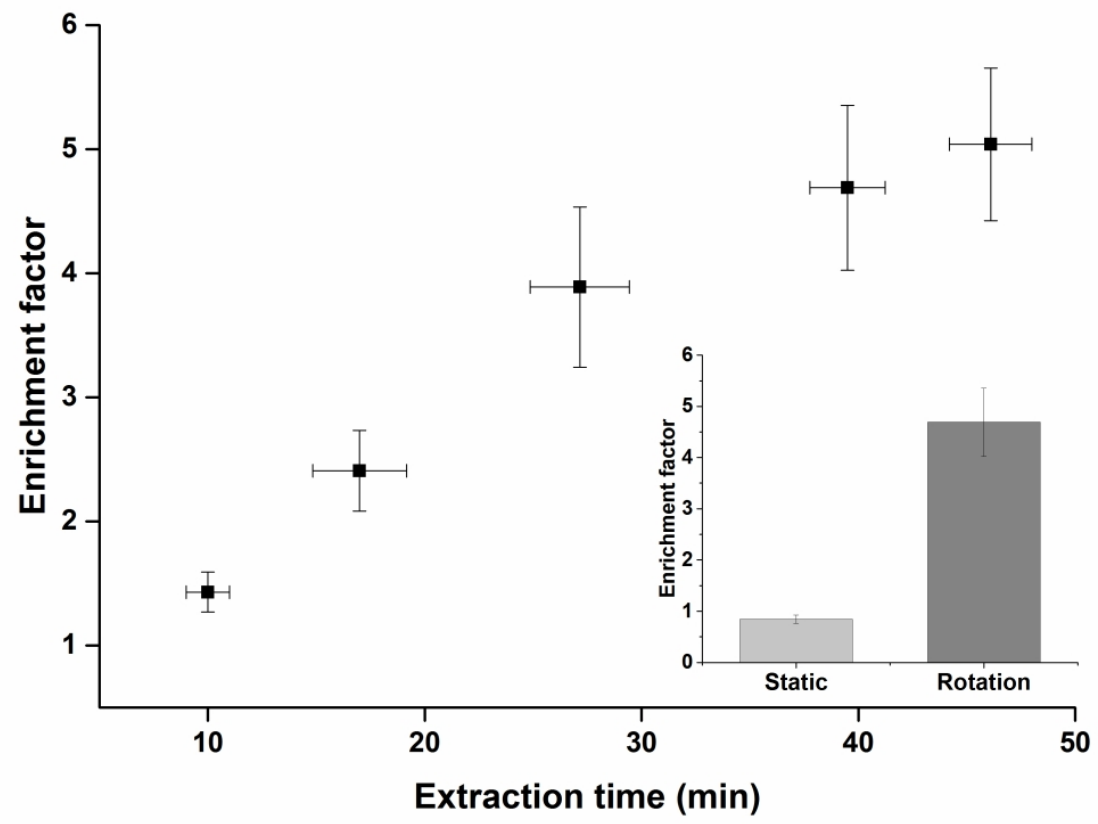

Figure 6: The enrichment obtained on-disc as function of extraction time. The standard deviation was calculated based on three repetitions. The enrichment under static condition and during extraction under rotation both cases with 40 min extraction time (inset).

$289 \times 202 \mathrm{~mm}(300 \times 300 \mathrm{DPI})$ 


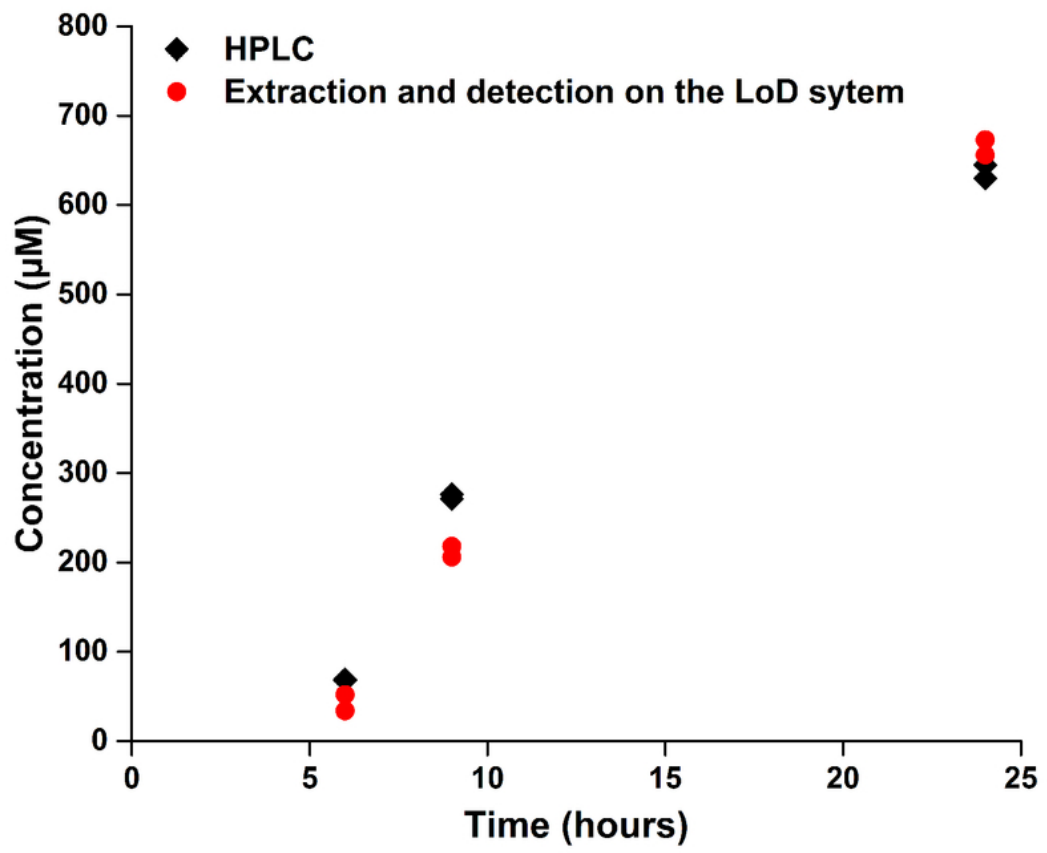

Figure 7: Quantification of pHCA concentration in samples from cell culture supernatant, obtained at 6, 9 and 24 hours, using the developed LoD device (red dots) and HPLC (black dots) $n=2$.

\section{$82 \times 63 \mathrm{~mm}(300 \times 300 \mathrm{DPI})$}



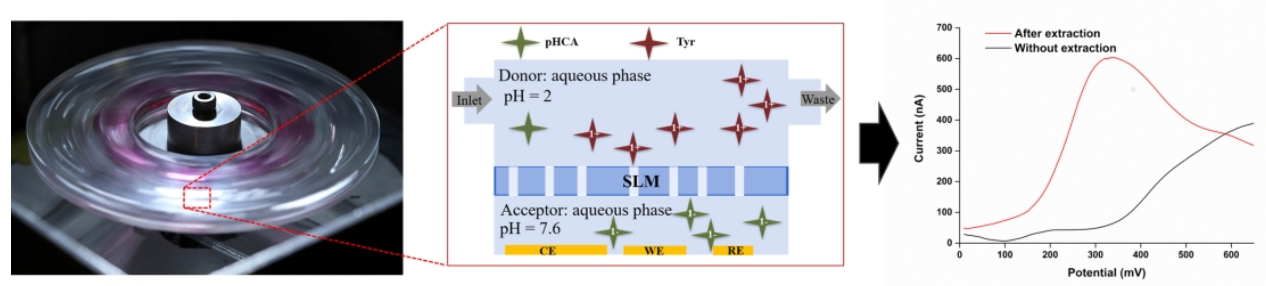

for TOC only

$205 \times 47 m m(300 \times 300$ DPI $)$ 